\title{
$\beta$-1,3-Glucan (schizophyllan) can act as a one-dimensional host for creation of novel poly(aniline) nano-fiber structures
}

Munenori Numata ${ }^{\dagger}$, Teruaki Hasegawa ${ }^{\dagger}$, Tomohisa Fujisawa ${ }^{\dagger}$, Kazuo Sakurai ${ }^{\ddagger}$, and Seiji Shinkai ${ }^{\dagger *}$

Department of Chemistry and Biochemistry, Graduate School of Engineering, Kyushu University, Fukuoka, 812-8581. Fax:+81-92-652-3611; Tel:+81-92-642-3585, and Department of Chemical Processes and Environments, Faculty of Environmental Engineering, The University of Kitakyushu, 1-1 Hibikino, Wakamatu-ku, Kitakyushu, Fukuoka, 808-0315.

\section{Supporting Information}

\section{General Procedures}

TEM and SEM observations were carried out using JEOL TEM-2010 (accelerate voltage 120 kV) and Hitachi S-5000, respectively. AFM observation was carried out using Topo METRIX SPM2100. CLSM images were taken using Carl Zeiss LSM510. CD and UV-VIS spectroscopic studies were performed on JASCO J-720WI and SHIMADZU UV-2500PC spectrophotometers, respectively.

\section{Materials}

SPG was kindly supplied by Taito Co., Japan. The molecular weight and the number of repeating units were evaluated to be $1.5 \times 10^{5}$ and 231 , respectively. Other polysaccharides used here were purchased from Tokyo Kasei Kougyou, Co. Poly(aniline) was obtained from Aldrich.

\section{Synthesis}

Mannose-modified SPG was synthesized according to a reported procedure. (reference 13a) 

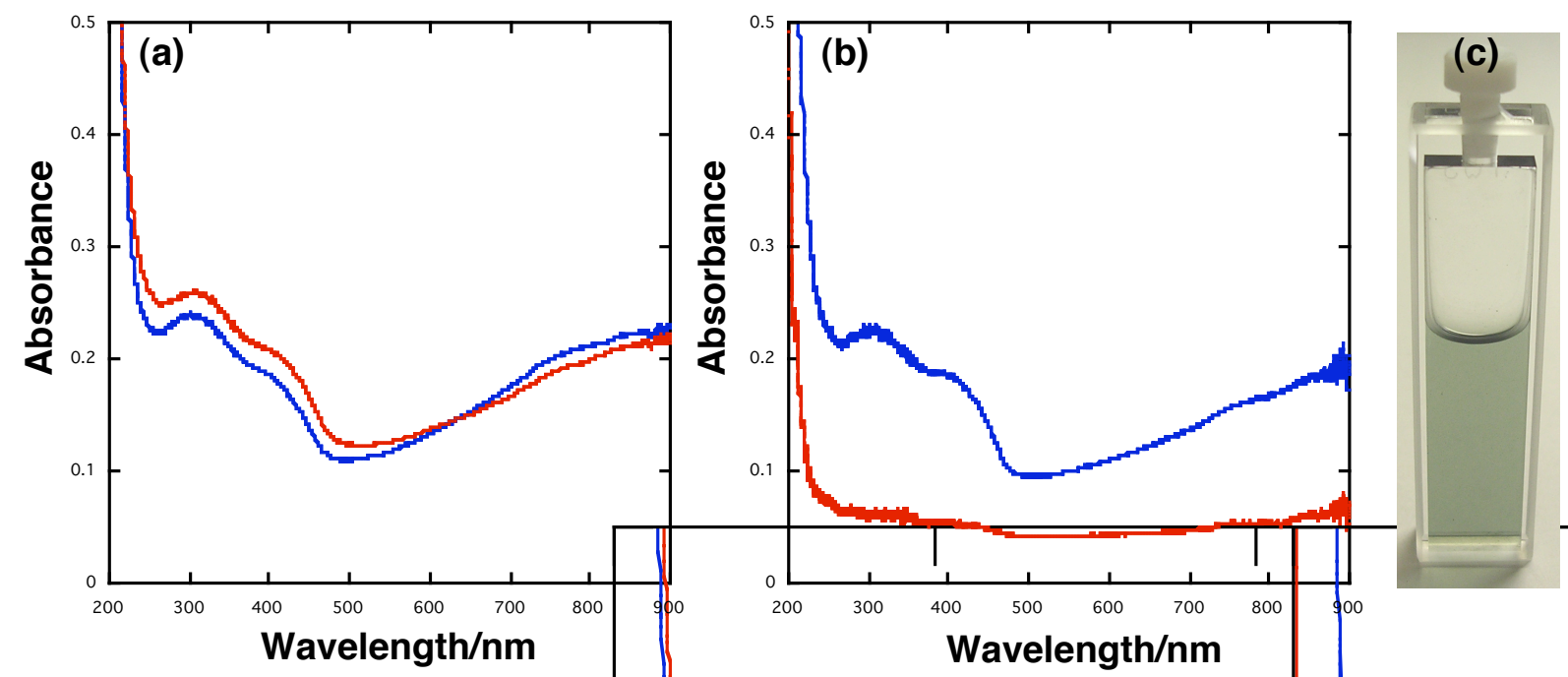

Figure S1. UV-VIS spectra of (a) SPG/PANIs composite (blue line) and amylose/PANls composite (red line) after $\mathrm{H}^{+}$doping, (b) after $24 \mathrm{~h}$ under the same conditions, the amylose/PANIs composite resulted in the precipitate (red line), and (c) photo image of the SPG-PANIs composite aqueous solution after $\mathrm{H}^{+}$doping, cell length $0.5 \mathrm{~cm}$.

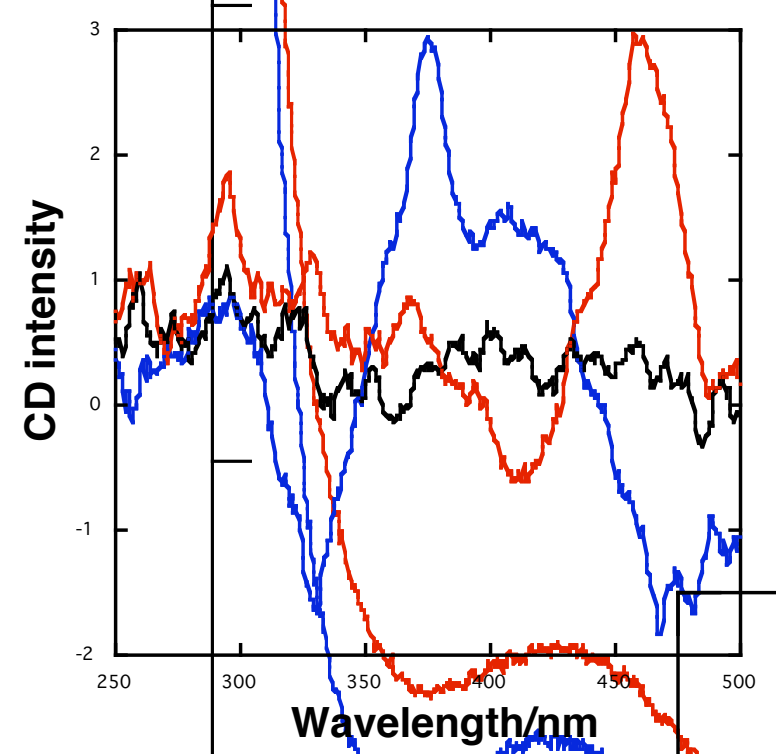

Figure S2. CD spectra of SPG/PANIs (blue line), amylose/PANIs (red line) and tSPG/PANIs mixture (black line), The CD spectrum for SPG/PANIs was very reproduceble whereas that for amylose/PANIs changed to some extent depending on the preparation procedure. 


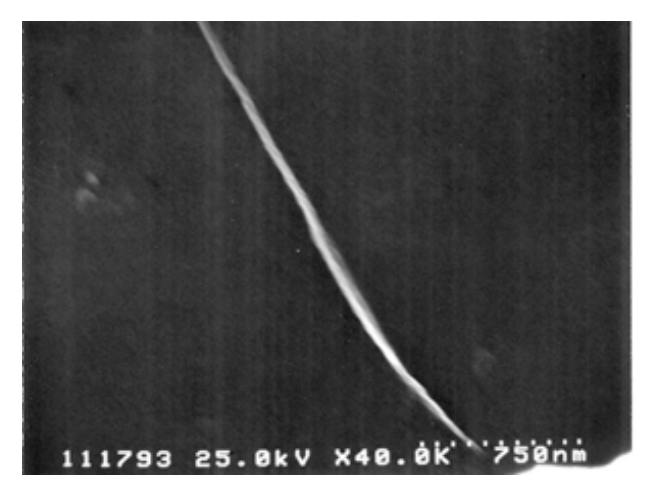

Figure S3. SEM image of the SPG/PANIs composite.
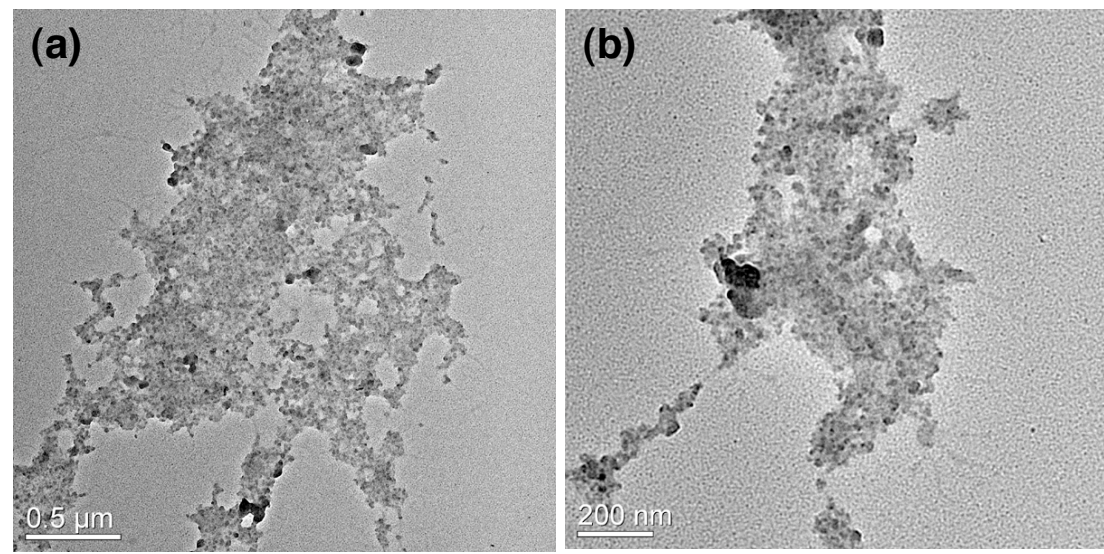

Figure S4. (a) TEM image of amylose/PANIs composite and (b) magnified image of (a), without staining.

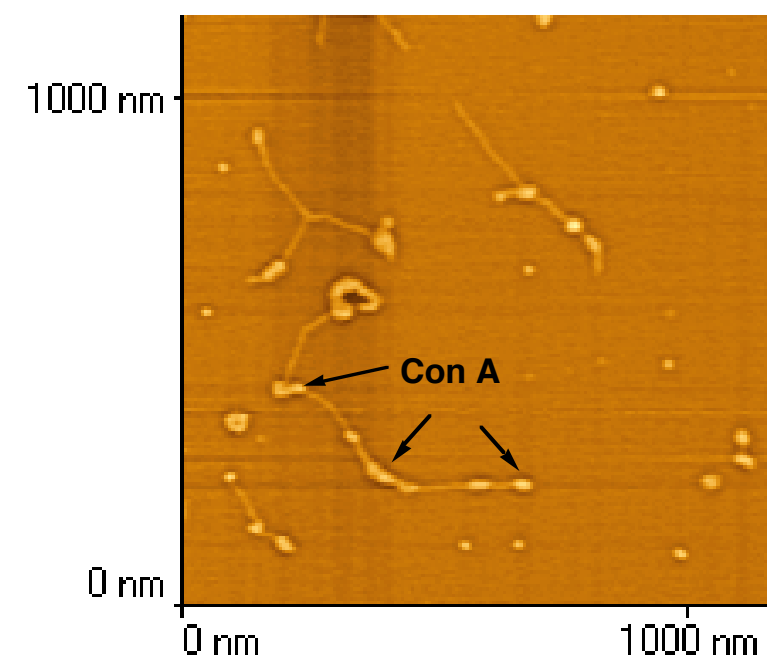

Figure S5. AFM image of the mannose-modified/PANIs composite + Con A. 